\title{
Transcriptional positive cofactor 4 promotes breast cancer proliferation and metastasis through c-Myc mediated Warburg effect
}

Peng Luo ${ }^{1 \dagger}$, Chi Zhang ${ }^{1 \dagger}$, Fengying Liao ${ }^{1}$, Long Chen ${ }^{1}$, Zhenyu Liu ${ }^{2}$, Lei Long ${ }^{1}$, Zhongyong Jiang ${ }^{1}$, Yawei Wang ${ }^{1}$, Ziwen Wang ${ }^{1}$, Zujuan Liu', Hongming Miao ${ }^{3}$ and Chunmeng Shi ${ }^{1 *}$ (D)

\begin{abstract}
Background: The human positive cofactor 4 (PC4) is initially identified as a transcriptional cofactor and has an important role in embryonic development and malignant transformation. However, the clinical significance and the molecular mechanisms of PC4 in breast cancer development and progression are still unknown.

Methods: We investigated PC4 expression in 114 cases of primary breast cancer and matched normal breast tissue specimens, and studied the impact of PC4 expression as well as the molecular mechanisms of this altered expression on breast cancer growth and metastasis both in vitro and in vivo.

Results: PC4 was significantly upregulated in breast cancer and high PC4 expression was positively correlated with metastasis and poor prognosis of patients. Gene set enrichment analysis (GSEA) demonstrated that the gene sets of cell proliferation and Epithelial-Mesenchymal Transition (EMT) were positively correlated with elevated PC4 expression. Consistently, loss of PC4 markedly inhibited the growth and metastasis of breast cancer both in vitro and in vivo. Mechanistically, PC4 exerted its oncogenic functions by directly binding to c-Myc promoters and inducing Warburg effect.
\end{abstract}

Conclusions: Our study reveals for the first time that PC4 promotes breast cancer progression by directly regulating c-Myc transcription to promote Warburg effect, implying a novel therapeutic target for breast cancer.

Keywords: Breast cancer, PC4, Warburg effect, C-Myc, Metastasis, Proliferation

\section{Background}

Breast cancer is one of the most common cancers and a leading cause of tumor-related death in females worldwide. Despite advances in treatments, many patients still develop into metastatic disease, which is the leading cause of breast cancer death [1-3]. Thus, there is an urgent need to characterize the underlying molecular mechanisms and identify novel therapeutic targets to improve the outcomes for breast cancer [4].

\footnotetext{
* Correspondence: shicm@tmmu.edu.cn

${ }^{\dagger}$ Peng Luo and Chi Zhang contributed equally to this work.

${ }^{1}$ Institute of Rocket Force Medicine, State Key Laboratory of Trauma, Burns and Combined Injury, Third Military Medical University, Chongqing 400038, China

Full list of author information is available at the end of the article
}

Recent studies have shown that metabolic reprogramming is a hallmark of cancer cells and a key contributor to cancer progression [5]. The Warburg effect [6], as known as aerobic glycolysis, is the best-characterized metabolic change in cancer cells, which facilitates cancer growth and progression by increasing glucose uptake, elevating lactate production, and supporting the energy demands [7]. Emerging evidence has indicated the crucial role of the Warburg effect in cancer therapy as a novel target [8]. In the process of glycolysis, the c-Myc oncogenic transcription factor plays a key role by directly regulating the glycolytic genes, such as GLUT1, HK2, LDHA, PDK1, etc. $[9,10]$. Although a few transcription factors have been reported to regulate glycolysis [11], the transcriptional regulation of Warburg effect

(C) The Author(s). 2019 Open Access This article is distributed under the terms of the Creative Commons Attribution 4.0 International License (http://creativecommons.org/licenses/by/4.0/), which permits unrestricted use, distribution, and 
and the upstream regulatory mechanism of c-Myc are still largely unknown.

The human positive cofactor 4 (PC4) and its yeast ortholog SUB1 (also named as coactivator p15) are initially identified as a coactivator of basal transcription $[12,13]$. PC4 is located on chromosome 5p13 and encodes a 127-amino acid protein that has an important role in various cellular processes including transcription [14-17], DNA replication [18-22], DNA repair [23-31] and chromatin organization [32, 33]. By the model of PC4 knockout mouse, we have found that loss of PC4 results in early embryo lethality, highlighting an essential role of PC4 in embryonic development [34]. And our previous studies have shown that overexpression of PC4 is involved in the malignant transformation of normal dermal multipotent fibroblasts, indicating the crucial role of PC4 in tumorigenesis [35]. Besides, PC4 is also found to be upregulated in lung cancer [36], astrocytoma [37], prostate cancer [38] and esophageal squmaous cell carcinoma [26] and positively related with tumor lymphatic metastasis [39] and chemo-radiosensitivity [26, 40, 41]. However, the clinical significance and the molecular mechanisms of PC4 in breast cancer development and progression are still unknown.

In this study, we reported firstly that PC4 was highly expressed in breast cancer and positively correlated with metastasis and poor prognosis of patients. Through gene set enrichment analysis (GSEA) in breast cancer specimens and experimental verification, we demonstrated that PC4 promoted the growth and metastasis of breast cancer both in vitro and in vivo. Furthermore, our findings revealed that PC4 exerted its oncogenic functions by directly binding to $\mathrm{c}-\mathrm{Myc}$ promoters and activating glycolysis. Therefore, our study provided novel insights into the functions and mechanisms of PC4 in breast cancer, and suggested that PC4 might be a promising therapeutic target for breast cancer.

\section{Methods}

\section{Cell lines and clinical samples}

The human breast cancer cell lines (MDA-MB-231 and MCF-7) were purchased from the American Type Culture Collection (ATCC, USA) and the Cell Bank of the Chinese (Shanghai, China). All cells were cultured in the recommended medium (Hyclone, USA), supplemented with 10\% FBS (Gibco, USA) and 1\% streptomycin/penicillin (Beyotime, Shanghai, China), and incubated in 5\% $\mathrm{CO}_{2}$ at $37^{\circ} \mathrm{C}$. A total of 114 paraffin-embedded breast cancer samples, with paired adjacent tissue and normal tissues were obtained at Southwest Hospital of Third Military Medical University. All breast cancer patients were diagnosed independently by at least two experienced pathologists, according to the Union for International Cancer Control classification system. The study was approved by the Ethics Committee of Third Military Medical University.

\section{Cell proliferation assay and colony formation assay}

Cell proliferation was measured with the Cell Counting Kit-8 (Dojindo, Japan). Cells were seeded into 96-well plates with a density of 2000 cells each well and $100 \mathrm{ul}$ medium. Cellular proliferation was measured according to the readout at the wavelength of $450 \mathrm{~nm}$. Data were read by a microplate reader (Multiskan Go Multimode Reader; Thermo Scientific). To determine their clonogenic ability, MDA-MB-231 and MCF-7 cells were trypsinized and seeded in 6-well plates. The medium was changed every three days, and cells were cultured for up to 14 day until colonies were clearly visible. At the endpoint, cells were washed twice with PBS, fixed with $4 \%$ paraformaldehyde, stained with crystal violet (Beyotime, China) for $30 \mathrm{~min}$, and then colonies with $>50$ cells were counted.

\section{Wound healing assay}

After transfection with the respective shRNAs, cells were tripsinized and seeded into 6-well plates to allow the cells to form a complete monolayer. After they became confluent, wounds were carefully introduced across the cell monolayer with a 10ul pipette tip. The dead cells were removed by PBS washes. Cells were treated with serum-free medium for $24 \mathrm{~h}$. Images were taken at the indicated time and then migration distance was measured.

\section{Transwell chamber assay}

Cell inserts pre-coated with matrigel $(8.0 \mu \mathrm{m}$ pore size membrane; Corning) were used. Breast cancer cells were deprived of serum for $12 \mathrm{~h}$ and seeded onto the upper chamber surface in serum-free medium at a density of $1 \times 10^{5}$ cells per well, and $20 \%$ FBS was placed in the lower chamber and incubated at $37^{\circ} \mathrm{C}$ for $24 \mathrm{~h}$. At the end of incubation, nonmotile cells on the upper surface of the filter were wiped off and the cells on the underside chamber were fixed with methanol and stained with Crystal Violet (Beyotime) and counted using bright-field microscopy in 5 random fields in triplicate inserts.

\section{ECAR, glucose uptake, lactate and ATP assays}

Extracellular acidification rate (ECAR) was measured using extracellular flux analyzer (XFp) analyzer (Seahorse Bioscience) as described by the manufacturer. Lactate production (Lactate Assay Kit) was measured as described by the manufacturer (BioVision). To measure glucose uptake, cells were incubated with a fluorescent $\mathrm{D}$-glucose derivative, 2-[N-(7-nitrobenz-2-oxa-1,3-diazo1-4-yl)amino] -2-deoxy-D-glucose (2-NBDG; APExBIO) for $20 \mathrm{~min}$ at $37^{\circ} \mathrm{C}$. The fluorescence intensity of 
2-NBDG was measured using by flow cytometry (BD FACSCanto $\left.\mathrm{II}^{\mathrm{mx}}\right)$, and data were analyzed with FlowJo software (Treestar). ATP production (Enhanced ATP Assay Kit) was measured according to the protocol recommended by manufacturer (Beyotime).

\section{RNA interference}

The shRNA targeting human PC4(shRNA1: 5' - GACA GGUGAGACUUCGAGATT -3'; 5' - UCUCGAAGUCU CACCUGUCTT -3'. shRNA2: 5' - ACAGAGCAGCAG CAGCAGATT - 3 '; $5^{\prime}$ - UCUGCUGCUGCUGCUCUGU TT $\left.-3^{\prime}\right)$ and negative control shRNA ( $5^{\prime}$-UUCUCCGAA CGUGUCACGUTT-3'; 5'-ACGUGACACGUUCGGAG AATT-3') were constructed by GenePharma (Shanghai, China). The human PC4 plasmid and control were purchased from GeneChem (Shanghai, China). The human c-Myc plasmid and control were purchased from GenePharma (Shanghai, China). According to the manufacturer's protocol, MDA-MD-231and MCF-7 cells were transfected with shRNA or plasmid according to the manufactures' instructions.

\section{Western blotting analysis}

MDA-MB-231 and MCF-7 cells were harvested, washed, and lysed with RIPA buffer (Beyotime, China) containing protease inhibitor cocktail (Roche) for $30 \mathrm{~min}$ on ice. Total protein was extracted, and quantitated by a BCA kit (Beyotime, China) according to the manufacturer's instruction. The protein samples were separated by electrophoresis in 10\% 12\% gel, and then transferred onto PVDF membranes (Millipore). Blotted membranes were blocked and incubation with primary antibodies overnight at $4{ }^{\circ} \mathrm{C}$. The membranes were washed $5 \mathrm{~min}$ for 3 times with TBST, and subsequently incubated $1 \mathrm{~h}$ with HRP-linked secondary antibody (Cell Signaling Technology, USA) at room temperature. The band intensities were visualized and detected by an enhanced chemiluminescence detection system (Bio-Rad Laboratories). Primary antibodies against c-Myc, E-cadherin, N-cadherin, Vimentin, GLUT1, LDHA and $\beta$-actin were obtained from Cell Signaling Technology. Primary antibodies against PC4 were obtained from Sigma.

\section{Quantitative RT-PCR}

Total RNA was extracted from breast cancer cells using Trizol (Cwbiotech, China). $1 \mu \mathrm{g}$ RNA was reverse transcribed into cDNA using the RevertAid First Strand cDNA Synthesis kit (\#K1622, Thermo Fisher Scientific,Inc.) according to the manufacturer's protocol. As described in our previous work, qRT-PCR was performed using a SYBR Green qPCR master mix (Takara) according to the manufacturer's protocol. After the reactions were completed, the comparative threshold cycle $(\mathrm{Ct})$ method was used to calculate the relative gene expression. GAPDH expression was used as the internal control. Human-specific primers were synthesized, and their sequences are shown in Additional file 1: Table S1.

\section{Chromatin immunoprecipitation (ChIP)}

MDA-MB-231 cells were conducted ChIP assay with the SimpleChIP Enzymatic Chromatin IP Kit (Magnetic Beads) (\#9003; Cell Signaling Technology) according to the manufacturer's instructions. Chromatin fragments were immunoprecipitated with anti-PC4 (NB10059774; Novus Biologicals), or rabbit IgG (\#2729; Cell Signaling Technology) coupled with ChIP Grade Protein G Magnetic Beads (\#9006; Cell Signaling Technology). After DNA purification, quantitative PCR was performed using primers (Additional file 1: Table S2). The fold enrichment was calculated by normalizing samples of anti-TCF-1 or p-STAT3 to normal rabbit IgG controls.

\section{Tumorigenicity assays}

Tumor model in vivo was established using 4-week-old female athymic nude mice from the Laboratory Animal Center of the Third Military Medical University (Chongqing, China) in a specific pathogen-free condition. Animal protocols were followed the Animal Care and Use Committee Guidelines of the Third Military Medical University. Mice were inoculated subcutaneously with $3 \times 10^{6}$ respective MDA-MB-231 cells in $100 \mathrm{ul} \mathrm{PBS}$ at one dorsal site. Tumor growth was grossly monitored and measured with sliding calipers every 2 days. Volume of tumors were calculated according to the formula: volume $\left(\mathrm{mm}^{3}\right)=\left(\right.$ width $^{2} \mathrm{X}$ length $) / 2$. When mice were killed, tumors were dissected and weighed. For the growth test, mice were sacrificed at the endpoint.

\section{Experimental metastasis assay}

Tumor metastasis assays were performed using an intravenous breast cancer mouse model as previous described [42]. Briefly, respective MDA-MB-231 cells were digested in PBS. Single-cell suspensions of $3 \times 10^{5}$ cells were injected into the tail vein of female NOD/SCID mice. Four weeks after injection, animals were sacrificed, lungs collected, and fixed in $10 \%$ buffered formalin for subsequent hematoxylin and eosin (H\&E) staining.

\section{Immunohistochemical staining}

The paraffin-embedded sections were dewaxed, rehydrated and incubated with human PC4 antibody (1: 500; Sigma, St. Louis, Missouri, USA) at $4{ }^{\circ} \mathrm{C}$ overnight. Subsequently, the slides were sequentially incubated with biotinylated secondary antibody at $37^{\circ} \mathrm{C}$ for $30 \mathrm{~min}$, and positive staining was visualized by using DAB. PC4 expression in breast cancer was evaluated by percentage of positive-staining cells and staining intensity. The percentage of positive cells was evaluated quantitatively and scored as: 0 ( $<5 \%$ positive tumor cells), 1 (5-25\% 
positive tumor cells), 2 ( $26-50 \%$ positive tumor cells), 3 (51-75\% positive tumor cells) and 4 ( $>75 \%$ positive tumor cells). Intensity was graded as follows: 0 , no signal; 1, weak (light yellow); 2, moderate (brown); and 3, strong staining. The final quantification of each staining was obtained by multiplying these 2 scores. A total staining score of $0-12$ was calculated and graded as negative $(-$, score $0-1)$, weak $(+$, score $2-4)$, moderate $(++$, score $5-8)$, or strong $(+++$, score 9-12). All tissue samples were examined and independently evaluated by two pathologists.

\section{Statistical analysis}

Statistical analysis was carried out using SPSS 13.0 software (SPSS Inc., Chicago, USA), and all data are presented as means $\pm \mathrm{SD}$. Comparisons between two groups were performed using the Student's t-test. Comparisons among three or more groups were performed using a one-way analysis of variance (ANOVA). The survival data was performed using the Kaplan-Meier method. Correlation between PC4 expression and clinical parameters was determined using the Pearson's $x^{2}$ method. $P<0.05$ indicated a statistically significant difference.

\section{Results}

PC4 is significantly upregulated in breast cancer and is closely correlated with metastasis and poor prognosis of patients

To explore the potential clinical significance of PC4, we firstly detected PC4 expression level in 114 cases of breast cancer patients compared with their adjacent counterparts. In both invasive ductal carcinoma (IDC) and invasive lobular carcinoma (ILC), aberrant PC4 overexpression was observed in carcinoma tissues from immunohistochemistry results, while weak positive signal was found in adjacent tissues and almost no positive signal in normal tissues (Fig. 1a). The average staining score of PC4 expression confirmed above results (Fig. 1b), implying a potential role of PC4 in breast cancer tumorigenesis. In addition, we analyzed PC4 expression in breast cancer with or without metastasis. As shown in Fig. 1c and d, the intensity of PC4 in carcinoma with metastasis was apparently higher than that without metastasis, suggesting a possible correlation between PC4 expression levels and progression of metastasis in breast cancer.

Meanwhile, through analyzing 3951 cases of breast cancer patients from public cancer databases (KaplanMeier plotter database), the higher PC4 expression group $(n=1976)$ had poorer overall survival compared with lower PC4 expression group $(n=1975)(P<0.0001$, Additional file 1: Figure S1). Data from Vant Veer breast cancer databases [43] and GSE9893 [44] also demonstrated that overall survival periods were shorter among patients with higher PC4 levels in the tumor $(P<0.05$,
Fig. 1e-f). Taken together, these results indicate that PC4 is commonly upregulated in breast cancer and may represent a significant molecular feature in breast cancer pathogenesis.

\section{PC4 knockdown suppresses breast cancer cell growth both in vitro and in vivo}

To investigate the functional significance of increased PC4 expression in breast cancer, we conducted gene set enrichment analysis (GSEA) to compare the gene expression profiles of $\mathrm{PC} 4^{\text {low }}$ and $\mathrm{PC} 4^{\text {high }}$ breast cancer specimens. GSE9893 dataset [44] containing 155 cases of breast cancer patients was divided into PC4 $4^{\text {low }}(n=78)$ and $\mathrm{PC} 4^{\text {high }}$ $(n=77)$ groups based on the median expression level of PC4. GSEA results demonstrated that the gene sets of Benporath_Proliferation were obviously enriched in PC4 ${ }^{\text {high }}$ breast cancer specimens, implying the potential role of $\mathrm{PC} 4$ in tumor proliferation $(\mathrm{P}<0.05$, Fig. $2 \mathrm{a})$. To verify the above results, MDA-MB-231 and MCF-7 cells were chosen for subsequent loss-of-function studies. The stable cell lines with PC4 knockdown were established by lentiviral infection (shPC4-1 and shPC4-2) (Fig. 2b). The CCK- 8 and colony formation assays demonstrated that silencing of PC4 dramatically reduced the proliferation (Fig. 2c) and colony formation capacity (Fig. 2d-e) of breast cancer cells. Besides, silencing of PC4 had no significant impact on cell apoptosis in both MDA-MB-231 and MCF-7 cells (Additional file 1: Figure S2).

Furthermore, we established a subcutaneous xenograft model to determine the effect of PC4 on breast cancer cell growth in vivo. The MDA-MB-231 cells with or without stable PC4-knockdown were inoculated subcutaneously into the right flank of female nude mice, and tumor sizes were measured every 2 days. During the whole experiment, the growth of xenografts in shPC4-1 and shPC4-2 group was inhibited compared with the control group (Fig. 2f). And the average tumor size and tumor weight at the endpoint was reduced by PC4 depletion (Fig. 2g-h). Taken together, these data suggest that PC4 promotes breast cancer cell growth both in vitro and in vivo.

\section{Loss of PC4 inhibits metastasis of breast cancer via reducing epithelial-mesenchymal transition both in vitro and in vivo}

Apart from the gene sets of proliferation, GSEA in GSE9893 also showed that the gene sets of HALLMARK_Epithelial_Mesenchymal_Transition were significantly enriched in $P C 4^{\text {high }}$ breast cancer samples $(P<0.001$, Fig. 3a). Owing to the crucial role of EMT in tumor metastasis [45], we conducted further experiments in vitro. The wound healing assays and transwell assays revealed that depletion of PC4 led to a significant inhibition of migration (Fig. 3d-e) and invasive capability (Fig. 3-c) in 


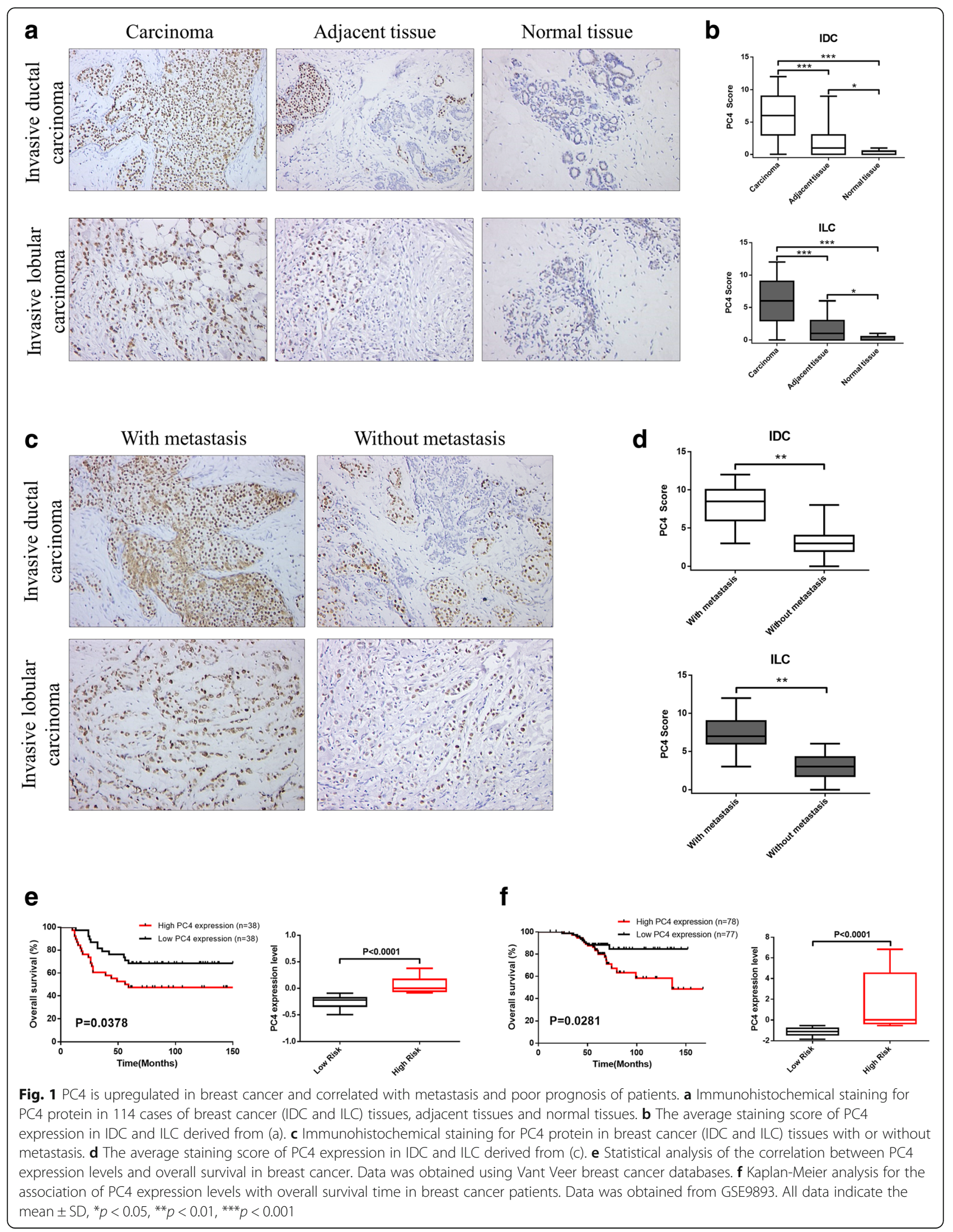



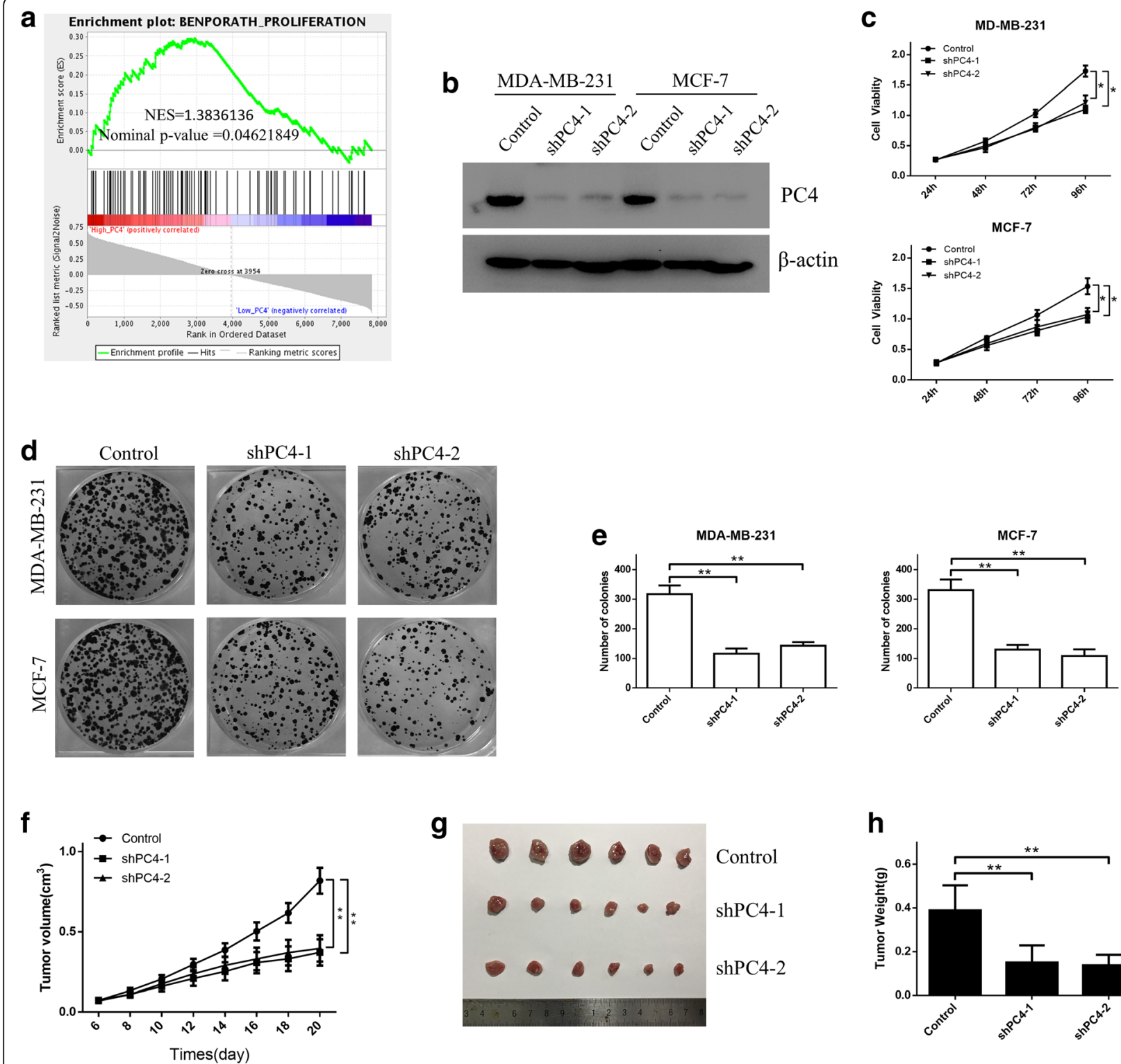

Fig. 2 PC4 knockdown suppresses breast cancer cell growth both in vitro and in vivo. a Gene Set Enrichment Analysis (GSEA) comparing the gene sets of Proliferation in PC4 ${ }^{\text {high }}(n=78)$ and PC4 ${ }^{\text {low }}(n=77)$ breast cancer patients. Data was obtained from GSE9893. NES means normalized enrichment score. $\mathbf{b}$ Two shRNAs (shPC4-1 and shPC4-2) were used to establish the stable PC4 knockdown cell lines (MDA-MB-231 and MCF-7 cells). The PC4 knockout efficiency were examined by western blot. c Cell viability of MDA-MB-231 and MCF-7 cells was measured using CCK-8 assay at $24 \mathrm{~h}, 48 \mathrm{~h}, 72 \mathrm{~h}$ and $96 \mathrm{~h}$ after PC4 knockdown. d The clone formation assay of MDA-MB-231 and MCF-7 cells after PC4 knockdown. e Statistical analysis of the data derived from (d). Experiments were repeated three times independently. $\mathbf{f}$ MDA-MB-231 cells with stable PC4knockdown were inoculated into female nude mice. Tumor growth curves were measured every 2 days, and the tumor volume was estimated using the following formula: volume $=$ length $\times$ width ${ }^{2} / 2$. $\mathbf{g}$ and $\mathbf{h}$ The dissected xenografts were photographed and weighed at the endpoint. All data indicate the mean $\pm \mathrm{SD} .{ }^{*} p<0.05,{ }^{* *} p<0.01$

both MDA-MB-231 and MCF-7 cells. Then, we detected the expression levels of EMT markers by western blot and qPCR. As shown in Fig. 3f and g, the protein and mRNA level of epithelial markers such as E-cadherin were increased, while the protein level of mesenchymal markers such as $\mathrm{N}$-cadherin and Vimentin were reduced in PC4-knockdown cells. These results demonstrate that
PC4 can promote cell migration and invasion and induce EMT in breast cancer cells.

Next, lung metastasis model, which was established by tail vein injection of PC4-knockdown and control breast cancer cells, was used to evaluate the role of PC4 in tumor metastasis in vivo. Female NOD/SCID mice injected with PC4-knockdown MDA-MB-231 cells 


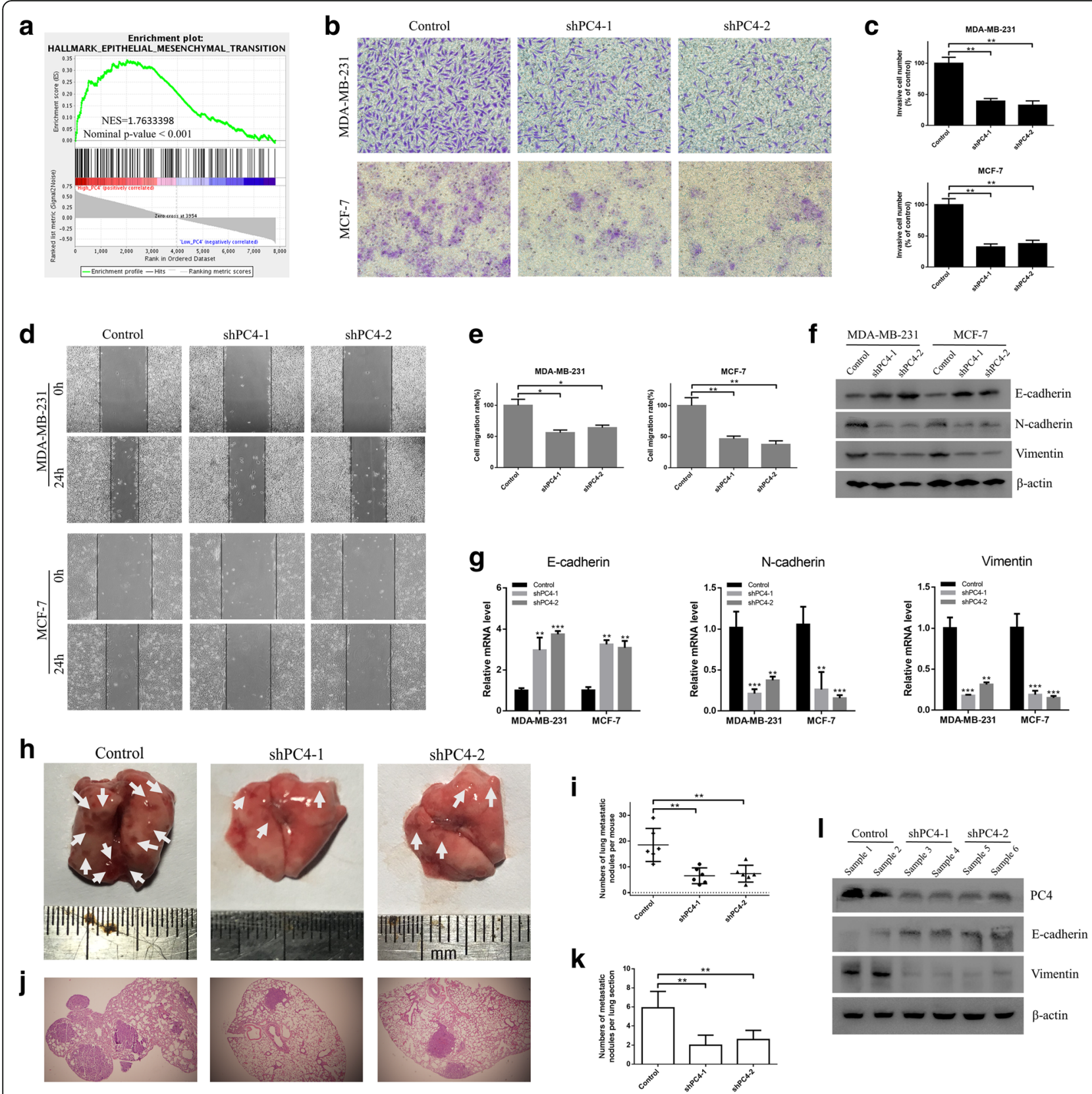

Fig. 3 Loss of PC4 inhibits metastasis of breast cancer cells in vitro and in vivo. a GSEA comparing the gene sets of Epithelial-Mesenchymal Transition (EMT) in PC4 ${ }^{\text {high }}(n=78)$ and PC4 ${ }^{\text {low }}(n=77)$ breast cancer patients. Data were obtained from GSE9893. NES means normalized enrichment score. b Cell invasive capacity was detected using the transwell assay after PC4 knockdown in MDA-MB-231 and MCF-7 cells. c Statistical analysis of the data derived from (c). Experiments were repeated three times independently. $\mathbf{d}$ Cell migration capacity was measured using the wound healing assay after PC4 knockdown in MDA-MB-231 and MCF-7 cells. e Statistical analysis of the data derived from (d). Experiments were repeated three times independently. $\mathbf{f}$ EMT markers (E-cadherin, N-cadherin and Vimentin) were detected by western blotting in MDA-MB-231 and MCF-7 cells after PC4 knockdown. $\mathbf{g}$ The mRNA levels of EMT markers (E-cadherin, N-cadherin and Vimentin) were detected by qPCR in MDA-MB-231 and MCF-7 cells after PC4 knockdown. $\mathbf{h}$ Tail vein injection of PC4-knockdown and control MDA-MB-231 cells in NOD/ SCID mice were used to establish lung metastasis model. Lung tissue was dissected and photographed at the endpoint. i Statistical analysis of lung metastatic lesions per mouse in PC4 knockdown groups and control group. $\mathbf{j}$ Hematoxylin and eosin (H\&E) staining of lung tissue in PC4 knockdown groups and control group. $\mathbf{k}$ Statistical analysis of lung metastatic nodules per lung section in PC4 knockdown groups and control group. I EMT markers (E-cadherin and Vimentin) were detected by western blot in dissected xenografts. All data indicate the mean $\pm S D$, ${ }^{*} p<$ $0.05,{ }^{* *} p<0.01,{ }^{* * *} p<0.001$. 
showed a significant decrease of lung metastatic lesions in comparison with control group (Fig. $3 \mathrm{~h}-\mathrm{i}$ ). And the hematoxylin and eosin ( $\mathrm{H} \& \mathrm{E})$ staining of lung tissues confirmed that depletion of PC4 resulted in greatly decreased lung metastasis of breast cancer cells in vivo (Fig. 3j-k). Moreover, Fig. 31 showed that downregulation of PC4 decreased the expression of Vimentin and increased the expression of E-cadherin, indicating that PC4 could induce EMT in vivo. Collectively, PC4 plays an important role in breast cancer metastasis via inducing EMT.

\section{Glycolysis is critically involved in the oncogenic functions of PC4}

To further illustrate the underlying mechanisms of PC4 mediated oncogenic advantages in breast cancer, GSEA in GSE9893 was conducted based on PC4 expression. As shown in Fig. 4a, the gene sets of HALLMARKS_GLYCOLYSIS were evidently enriched in $\mathrm{PC}_{4}^{\text {high }}$ breast cancer samples $(P<0.05)$, indicating that glycolysis activity was positively correlated with high expression of PC4 in breast cancer. To our knowledge, Warburg effect, also known as aerobic glycolysis, is a remarkable hallmark of cancer, which provides the main source of energy and maintains the malignant phenotypes of cancer cells [46]. Thus, we tested whether PC4 could modulate the glycolytic phenotype in breast cancer cells. As expected, PC4 knockdown decreased glucose uptake (Fig. 4b), lactate production (Fig. 4c) and ATP generation (Fig. 4d) in MDA-MB-231 cells. PC4 knockdown also inhibited extracellular acidification rate (ECAR), which indicated overall glycolytic flux (Fig. 4e-h). Moreover, loss of PC4 inhibited the expression of GLUT1 and LDHA (Fig. 4i), the key enzymes of glycolysis. Hence, PC4 plays an essential role in maintaining Warburg effect in breast cancer cells.

Owing to the important role of Warburg effect in cancer development and progression [7], we next confirmed whether the oncogenic functions of PC4 were dependent on glycolysis. The glycolytic inhibitor 2-deoxy-D-glucose (2-DG) was used to block glycolysis in MDA-MB-231 cells, and the specific plasmid was used to overexpress PC4 before 2-DG treatment (Fig. 4k). Then, we found that overexpression of $\mathrm{PC} 4$ increased the proliferation (Fig. 4o), migration (Fig. 4j-l) and invasion (Fig. 4m-n) of breast cancer cells, which was inhibited by 2-DG. These results demonstrate that $\mathrm{PC} 4$ exerts its oncogenic functions by promoting glycolysis in breast cancer.

\section{PC4 promotes glycolysis by regulating c-Myc transcription}

Considering that c-Myc is a critical regulator of glycolysis in cancers by directly transactivating glycolytic genes [47], we performed GSEA in GSE9893 to explore the possible correlations between c-Myc and PC4. Interestingly, the gene sets of HALLMARKS_MYC_TARGETS_V1 were markedly enriched in $\mathrm{PC}_{4}^{\text {high }}$ breast cancer samples (Fig. 5a, $P<0.001$ ), indicating that c-Myc was positively correlated with high expression of PC4 in breast cancer. The qPCR (Fig. 5b) and western blot results (Fig. 5c) also showed that knockdown of PC4 observably downregulated the expression of $\mathrm{c}-\mathrm{Myc}$ in both MDA-MB-231 and MCF-7 cells. Immunohistochemistry staining in nude mice xenografts (Additional file 1: Figure S3) also confirmed that loss of PC4 could decrease the expression of c-Myc in vivo. To further demonstrate whether $\mathrm{c}-\mathrm{Myc}$ was responsible for the oncogenic functions of $\mathrm{PC} 4$, we used the specific plasmid to overexpress of c-Myc in PC4-knockdown cells (Fig. 5k). As expected, c-Myc overexpression rescued the decreased glucose uptake (Fig. 5h), lactate production (Fig. 5i) and ATP generation (Fig. 5j) in PC4-knockdown MDA-MB-231 cells. Overexpression of c-Myc also reversed extracellular acidification rate (ECAR) loss in PC4-knockdown cells (Fig. 5d-g). Moreover, the inhibitory effects of PC4 knockdown on proliferation (Fig. 5p), migration (Fig. 5l-m) and invasion (Fig. 5n-o) were also rescued by c-Myc overexpression. At last, the downregulation of GLUT1, LDHA, Vimentin and upregulation of E-cadherin in PC4-knockdown cells were reversed by overexpression of c-Myc (Fig. 5k). Collectively, these results suggest that $\mathrm{PC} 4$ exerts its oncogenic functions via activating c-Myc mediated glycolysis.

Given the important role of PC4 in transcription regulation [19], we subsequently examined whether PC4 could directly activate c-Myc transcription to promote its expression. To confirm the relationship between PC4 and c-Myc, we performed a bioinformatic analysis for the presence of putative PC4-binding sites (BS) in the C-MYC promoter, and found two putative $\mathrm{PC} 4 \mathrm{BS}$ in a region encompassing $0.1 \mathrm{~kb}$ upstream the transcription start site (TSS). Chromatin Immunoprecipitation (ChIP) showed that PC4 binds to the C-MYC promoter in that region (Fig. 5q), indicating that C-MYC is a direct downstream target of PC4.

\section{Discussion}

Recurrence and metastasis are the two leading causes of death in breast cancer, especially in Oestrogen receptor (ER), progesterone receptor (PR) and human epidermal growth factor receptor 2 (HER2) triple-negative breast cancer (TNBC) [48]. Clarifying the underlying mechanisms of TNBC progression and developing novel therapeutic targets are crucial for breast cancer treatment $[49,50]$. Here, we report a novel oncogene, PC4, which plays an important role in both ER-positive MCF-7 and triple-negative MDA-MB-231 breast cancer 

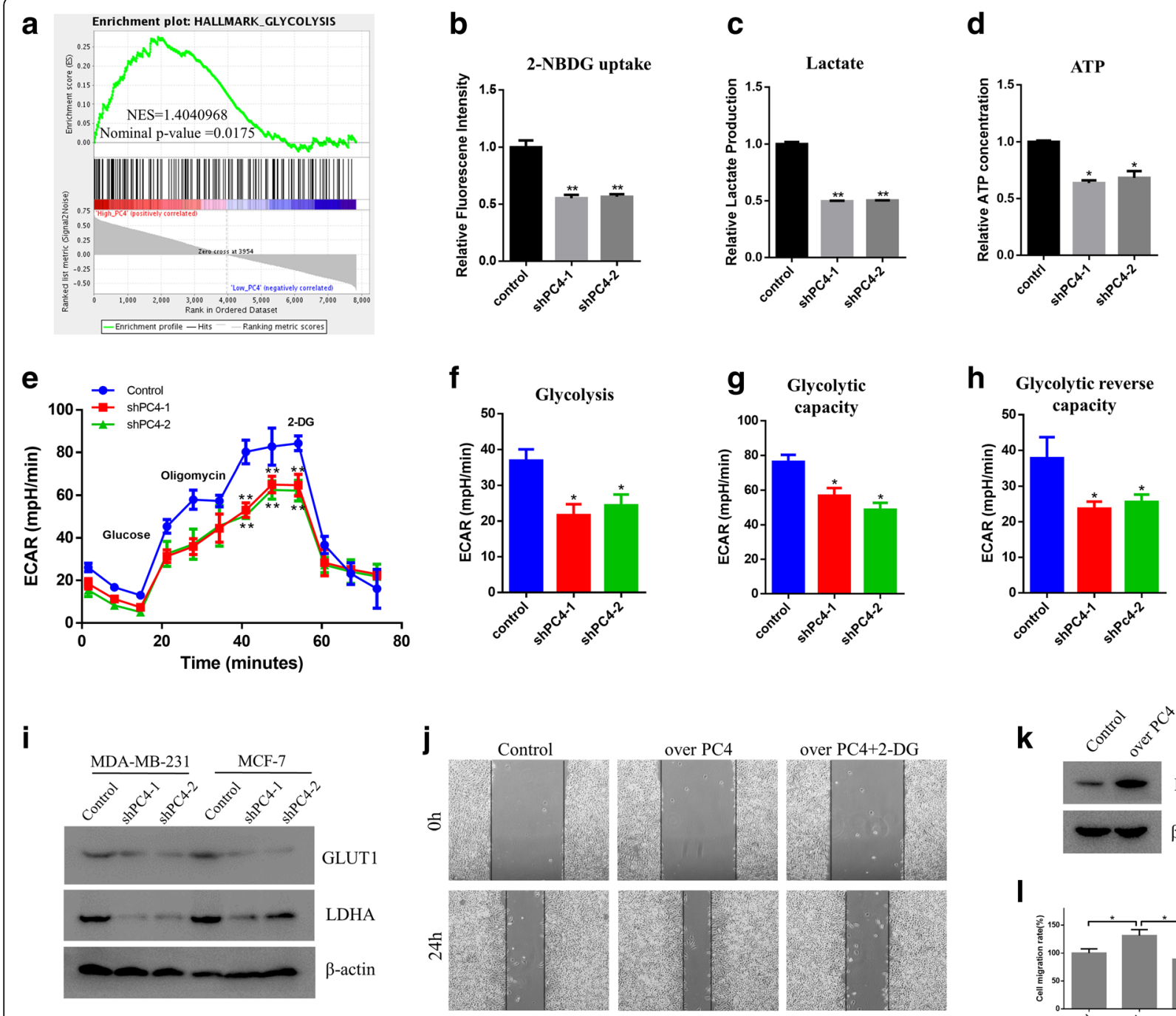

m

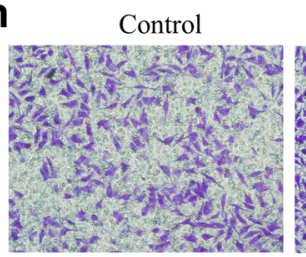

over PC4

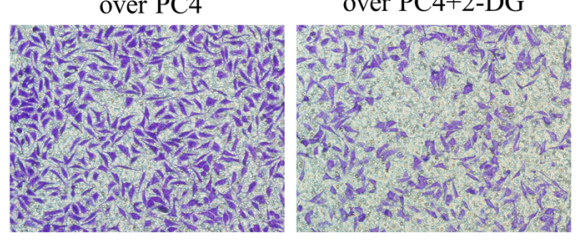

n

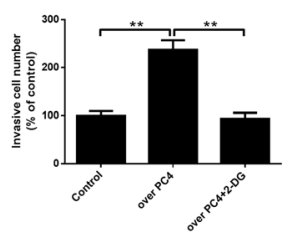

$\mathbf{k}$

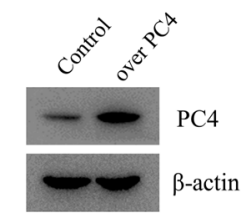

I

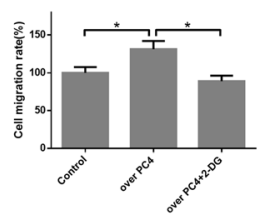

O

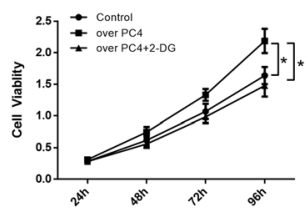

Fig. 4 Glycolysis is critically involved in the oncogenic functional of PC4. a GSEA comparing the gene sets of glycolysis in PC4 ${ }^{\text {high }}(n=78)$ and PC4 ${ }^{\text {low }}(n=77)$ breast cancer patients. Data was obtained from GSE9893. NES means normalized enrichment score. $\mathbf{b}, \mathbf{c}$ and $\mathbf{d}$ Glucose uptake, the production of lactate and ATP were determined in PC4 knockdown MDA-MB-231 cells and control cells. e, $\mathbf{f}, \mathbf{g}$ and $\mathbf{h}$ The extracellular acidification rate (ECAR) was determined in PC4 knockdown MDA-MB-231 cells and control cells. i Glycolysis markers (GLUT1 and LDHA) were detected by western blotting in MDA-MB-231 and MCF-7 cells after PC4 knockdown. $\mathbf{j}$ Cell migration capacity was measured using the wound healing assay after 2-DG treatment in PC4 overexpression MDA-MB-231 cells. $\mathbf{k}$ The specific plasmid was used to overexpress of PC4 and the protein level of PC4 was examined by western blot. I Statistical analysis of the data derived from (j). Experiments were repeated three times independently. $\mathbf{m}$ Cell invasive capacity was detected using the transwell assay in control cells, PC4 overexpression cells and 2-DG treated PC4 overexpression MDA-MB-231 cells. $\mathbf{n}$ Statistical analysis of the data derived from $\mathrm{m}$. Experiments were repeated three times independently. $\mathbf{o}$ Cell viability was measured by CCK-8 assay in control cells, PC4 overexpression cells and 2-DG treated PC4 overexpression MDA-MB-231 cells. All data indicate the mean $\pm \mathrm{SD},{ }^{*} p<0.05,{ }^{* *} p<0.01$ 


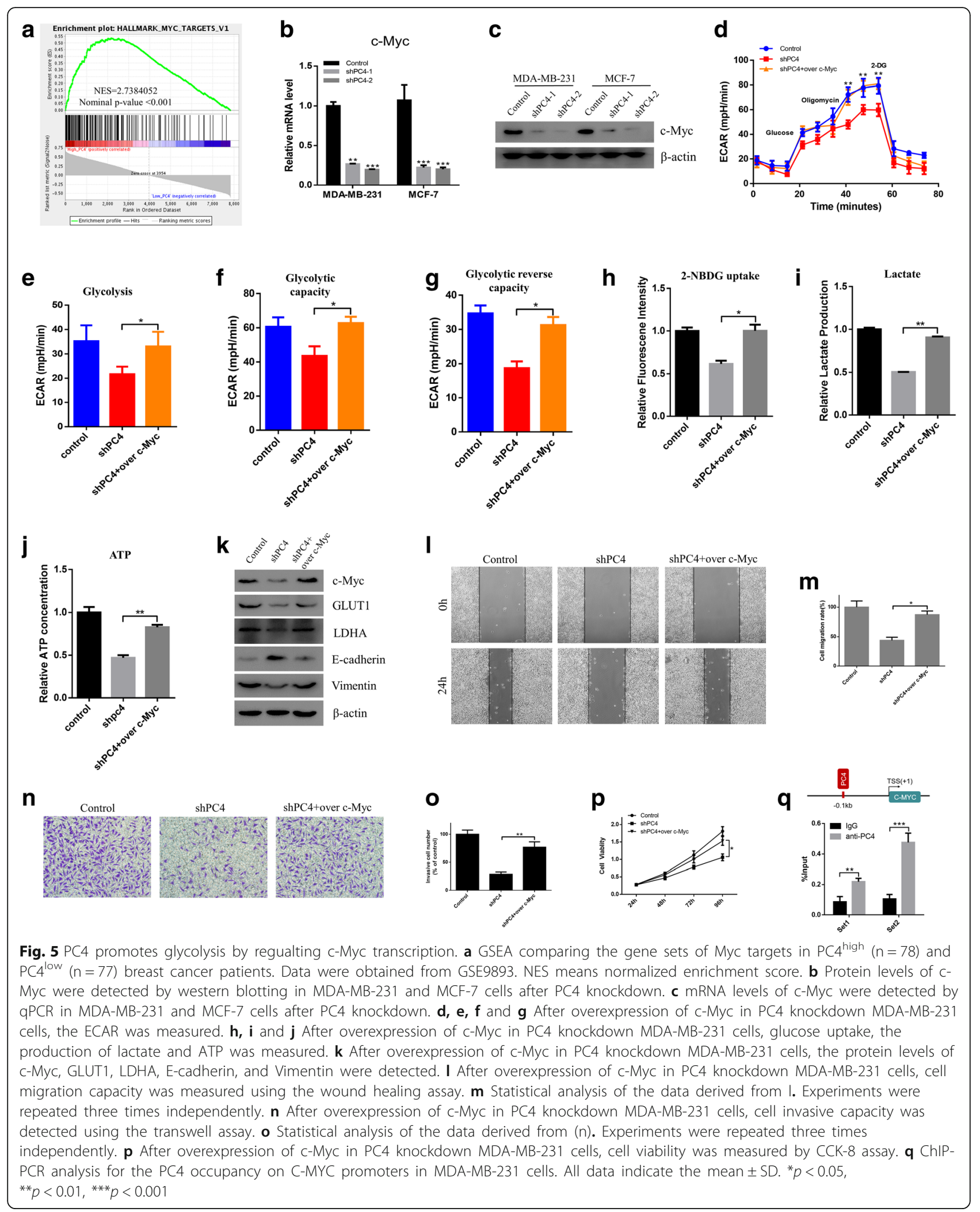


cell growth and metastasis by enhancing c-Myc mediated Warburg effect.

PC4 is initially identified as a transcription coactivator and contains highly conserved sequence among human, rat, mouse and yeast $[12,13]$. As a nuclear protein, PC4 plays important roles in various cellular processes including basal transcription [14-17], DNA replication [18-22], DNA repair [23-31] and chromatin organization [32, 33]. More specifically, PC4 interacts with distinct domains of transcription activators such as GAL4-VP16 [51], AP2 [52] and SMYD3 [22] to regulate their functions. Based on the studies of its biological function, we have reported that PC4 was significantly upregulated in the malignant transformation of normal dermal multipotent fibroblasts, implying the crucial role of PC4 in tumorigenesis [35]. We and other researchers also showed that PC4 was highly expressed in several tumors [36-38], and regulated lymphatic metastasis [39] and chemo-radiosensitivity [26, 40,41]. Although some studies showed the potential oncogenic role of PC4 in tumor, the moleculer mechanisms of PC4 in tumorigenesis and cancer progression are still unclear, especially in breast cancer. In this study, we specifically characterized PC4 expression in breast cancer. From immunohistochemistry results in both IDC and ILC, PC4 was demonstrated to be significantly upregulated and closely correlated with metastasis. The Kaplan-meier analysis of clinical data showed that PC4 was a potential predictor of poor prognosis in breast cancer. These findings led us to further explore the functional significance of increased PC4 expression in breast cancer. Through GSEA on 155 cases of breast cancer patients, we found that the gene sets of proliferation and EMT were positively correlated with high expression of PC4 in breast cancer. In both in vitro and in vivo experiments, PC4 knock-down obviously inhibited the growth and metastasis of breast cancer. Taken together, PC4 may be a promising therapeutic target in breast cancer.

As a hallmark of cancer cells, metabolic reprogramming is a key process during tumorigenesis and cancer progression [53]. Cancer cells frequently exhibit high rates of aerobic glycolysis, which facilitates cancer growth and progression by increasing glucose uptake, elevating lactate production, and supporting the energy demands [54]. In the process of glycolysis, c-Myc plays a key role by directly regulating the glycolytic enzyme genes [55]. By GSEA, we found that glycolysis-related genes and c-Myc target genes were positively correlated with high expression of PC4 in breast cancer. These findings led us to explore whether the oncogenic functions of PC4 were dependent on c-Myc mediated glycolysis. Indeed, PC4 knock-down dramatically inhibited the expression of c-Myc, as well as glucose uptake, lactate production, ATP generation, ECAR and the protein level of GLUT1 and LDHA. And Overexpression of c-Myc could reverse the decreased glycolysis and malignant phenotypes in PC4-knockdown cells, implying that PC4 exerted its oncogenic roles by c-Myc mediated glycolysis. Finally, the ChIP assays revealed that PC4 could directly bind to $\mathrm{c}-\mathrm{Myc}$ promoters and transcriptionally activate c-Myc to promote its expression. Given the central role of c-Myc in many human cancers especially in

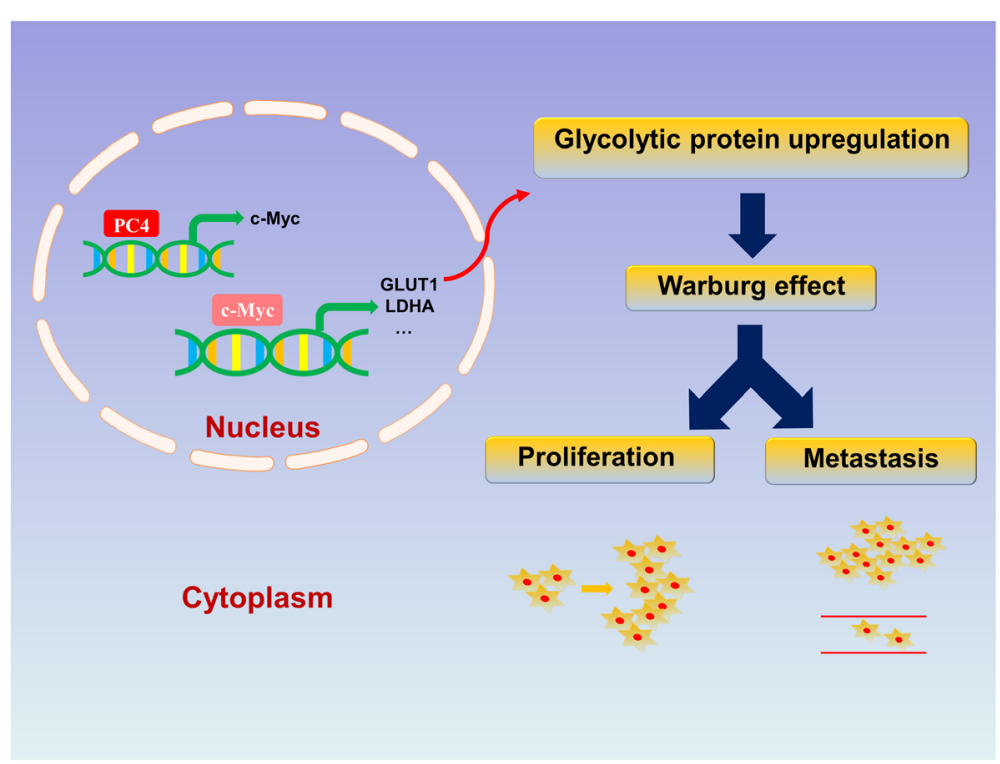

Fig. 6 Possible Mechanism of PC4 mediated promotion of breast cancer proliferation and metastasis. Schematic representation of PC4 oncogenic functions in breast cancer cells. PC4 exerts its oncogenic functions by directly binding to c-Myc promoters and inducing Warburg effect to promote tumor growth and metastasis 
triple negative breast cancer [56], c-Myc remains a promising target for effective antitumor therapy [57]. Unfortunately, c-Myc presents specific, significant obstacles to develop a strategy for its direct inhibition [57]. Thus, it is critical that the c-Myc transcription regulation be well-understood, particularly in its role in cancer progression and development.

\section{Conclusion}

This study is the first report of the expression pattern, diagnostic and prognostic value and oncogenic roles of PC4 in breast cancer. Furthermore, as a newly identified upstream regulator of c-Myc in breast cancer, PC4 exerts its oncogenic functions by directly binding to c-Myc promoters and inducing Warburg effect (Fig. 6). Therefore, our study provides novel insights into the functions and mechanisms of PC4 in breast cancer, and suggest that PC4 may be a novel therapeutic target for breast cancer.

\section{Additional file}

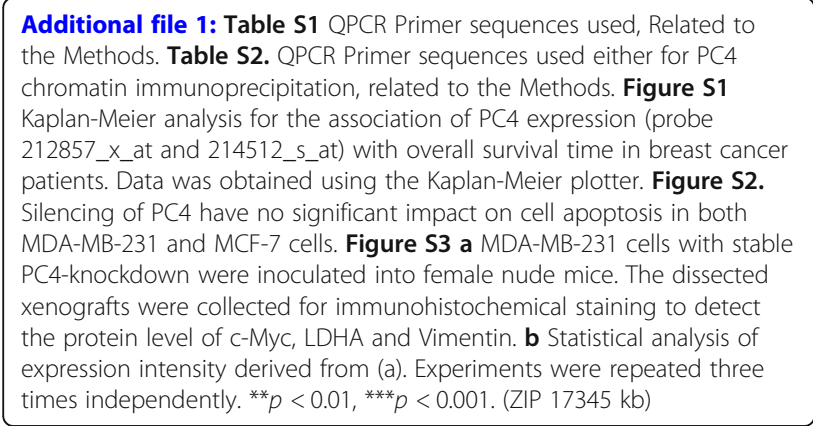

\section{Abbreviations}

2-DG: 2-deoxy-D-glucose; CCK8: Cell counting kit-8; ChIP: Chromatin immunoprecipitation; ECAR: Extracellular acidification rate; EMT: EpithelialMesenchymal Transition; GSEA: Gene set enrichment analysis; IDC: invasive ductal carcinoma; ILC: Invasive lobular carcinoma; PC4: Positive cofactor 4; qPCR: quantitative polymerase chain reaction

\section{Acknowledgements}

Not applicable.

\section{Publisher'sNote}

Springer Nature remains neutral with regard to jurisdictional claims in published maps and institutional affiliations.

\section{Funding}

This work was supported by National Key Research and Development Program (2016YFC1000805), Natural Science Foundation Programs (81372727 and 81773352) and University Innovation Team Building Program of Chongqing (CXTDG201602020).

\section{Availability of data and materials}

The data regarding to the expression of PC4 in breast cancer were from an open database (https://www.ncbi.nlm.nih.gov/geo/query/acc.cgi?acc= GSE9893).

\section{Authors' contributions}

$\mathrm{PL}$ and $\mathrm{CZ}$ performed the project, analyzed research data, and wrote the manuscript. FL, LC and ZL took part in animal experiments and immunohistochemistry. YW, ZW and ZJ participated in cellular and molecular biology experiments (RNAi, qPCR, western blotting analysis and ChIP). LL and $\mathrm{ZL}$ contributed to the collection of human tissues. HM contributed to helpful discussion and reviewed the manuscript. CS designed the study, supervised the experiments and revised the manuscript. All authors have read and approved the final manuscript.

\section{Ethics approval}

All the mouse experiments were approved by the Institutional Animal Care and Use Committee of the Third Military Medical University and carried out in accordance with the "Guide for the care and use of laboratory animals" published by the US National Institutes of Health (Publication no.85-23, revised 1996).

\section{Consent for publication \\ Not applicable.}

\section{Competing interests}

The authors declare that they have no competing interests.

\section{Publisher's Note}

Springer Nature remains neutral with regard to jurisdictional claims in published maps and institutional affiliations.

\section{Author details}

${ }^{1}$ Institute of Rocket Force Medicine, State Key Laboratory of Trauma, Burns and Combined Injury, Third Military Medical University, Chongqing 400038, China. ${ }^{2}$ Institute of Immunology, Third Military Medical University, Chongqing 400038, China. ${ }^{3}$ Department of Biochemistry and Molecular Biology, Third Military Medical University, Chongqing 400038, China.

Received: 10 January 2019 Accepted: 3 April 2019

Published online: 16 April 2019

\section{References}

1. Bray F, Ferlay J, Soerjomataram I, Siegel RL, Torre LA, Jemal A. Global cancer statistics 2018: GLOBOCAN estimates of incidence and mortality worldwide for 36 cancers in 185 countries. CA Cancer J Clin. 2018;68:394-424.

2. DeSantis C, Ma J, Bryan L, Jemal A. Breast cancer statistics, 2013. CA Cancer J Clin. 2014;64:52-62

3. Siegel RL, Miller KD, Jemal A. Cancer statistics, 2018. CA Cancer J Clin. 2018; 68:7-30.

4. Denkert C, Liedtke C, Tutt A, von Minckwitz G. Molecular alterations in triple-negative breast cancer-the road to new treatment strategies. Lancet. 2017;389:2430-42.

5. Hanahan D, Weinberg RA. Hallmarks of cancer: the next generation. Cell. 2011;144:646-74.

6. Warburg O, Wind F, Negelein E. The metabolism of tumors in the body. J Gen Physiol. 1927:8:519-30.

7. Koppenol WH, Bounds PL, Dang CV. Otto Warburg's contributions to current concepts of cancer metabolism. Nat Rev Cancer. 2011;11:325-37.

8. Vander Heiden MG. Targeting cancer metabolism: a therapeutic window opens. Nat Rev Drug Discov. 2011;10:671-84.

9. Dang CV. MYC on the path to cancer. Cell. 2012;149:22-35.

10. Dang CV, Le A, Gao P. MYC-induced cancer cell energy metabolism and therapeutic opportunities. Clin Cancer Res. 2009;15:6479-83.

11. Li L, Liang Y, Kang L, Liu Y, Gao S, Chen S, Li Y, You W, Dong Q, Hong T, et al. Transcriptional regulation of the Warburg effect in Cancer by SIX1. Cancer Cell. 2018:33:368-385 e367.

12. Ge H, Roeder RG. Purification, cloning, and characterization of a human coactivator, PC4, that mediates transcriptional activation of class II genes. Cell. 1994;78:513-23.

13. Kretzschmar M, Kaiser K, Lottspeich F, Meisterernst M. A novel mediator of class II gene transcription with homology to viral immediate-early transcriptional regulators. Cell. 1994;78:525-34.

14. Malik S, Guermah M, Roeder RG. A dynamic model for PC4 coactivator function in RNA polymerase II transcription. Proc Natl Acad Sci U S A. 1998; 95:2192-7.

15. Wang Z, Roeder RG. DNA topoisomerase I and PC4 can interact with human TFIIIC to promote both accurate termination and transcription reinitiation by RNA polymerase III. Mol Cell. 1998;1:749-57. 
16. Calvo O, Manley JL. The transcriptional coactivator PC4/Sub1 has multiple functions in RNA polymerase II transcription. EMBO J. 2005;24:1009-20.

17. Tavenet A, Suleau A, Dubreuil G, Ferrari R, Ducrot C, Michaut M, Aude JC, Dieci G, Lefebvre O, Conesa C, Acker J. Genome-wide location analysis reveals a role for Sub1 in RNA polymerase III transcription. Proc Natl Acad Sci U S A. 2009:106:14265-70.

18. Akimoto Y, Yamamoto S, lida S, Hirose Y, Tanaka A, Hanaoka F, Ohkuma Y. Transcription cofactor PC4 plays essential roles in collaboration with the small subunit of general transcription factor TFIIE. Genes Cells. 2014;19:879-90.

19. Garavis M, Calvo O. Sub1/PC4, a multifaceted factor: from transcription to genome stability. Curr Genet. 2017;63:1023-35.

20. Garavis M, Gonzalez-Polo N, Allepuz-Fuster P, Louro JA, Fernandez-Tornero C, Calvo O. Sub1 contacts the RNA polymerase II stalk to modulate mRNA synthesis. Nucleic Acids Res. 2017;45:2458-71.

21. Jo J, Hwang S, Kim HJ, Hong S, Lee JE, Lee SG, Baek A, Han H, Lee Jl, Lee I, Lee DR. An integrated systems biology approach identifies positive cofactor 4 as a factor that increases reprogramming efficiency. Nucleic Acids Res. 2016:44:1203-15

22. Kim JM, Kim K, Schmidt T, Punj V, Tucker H, Rice JC, Ulmer TS, An W: cooperation between SMYD3 and PC4 drives a distinct transcriptional program in cancer cells. Nucleic Acids Res 2015, 43:8868-8883.

23. Gao J, Zybailov BL, Byrd AK, Griffin WC, Chib S, Mackintosh SG, Tackett AJ, Raney KD. Yeast transcription co-activator Sub1 and its human homolog PC4 preferentially bind to G-quadruplex DNA. Chem Commun (Camb). 2015;51:7242-4

24. Huang J, Zhao Y, Liu H, Huang D, Cheng X, Zhao W, Taylor IA, Liu J, Peng YL. Substitution of tryptophan 89 with tyrosine switches the DNA binding mode of PC4. Sci Rep. 2015:5:8789.

25. Mortusewicz O, Evers B, Helleday T. PC4 promotes genome stability and DNA repair through binding of ssDNA at DNA damage sites. Oncogene. 2016;35:761-70.

26. Qian D, Zhang B, Zeng XL, Le Blanc JM, Guo YH, Xue C, Jiang C, Wang HH, Zhao TS, Meng MB, et al. Inhibition of human positive cofactor 4 radiosensitizes human esophageal squmaous cell carcinoma cells by suppressing XLF-mediated nonhomologous end joining. Cell Death Dis. 2014;5:e1461.

27. Yu L, Ma H, Ji X, Volkert MR. The Sub1 nuclear protein protects DNA from oxidative damage. Mol Cell Biochem. 2016;412:165-71.

28. Yu L, Volkert MR. Differential requirement for SUB1 in chromosomal and plasmid double-strand DNA break repair. PLoS One. 2013;8:e58015.

29. Lopez CR, Singh S, Hambarde S, Griffin WC, Gao J, Chib S, Yu Y, Ira G, Raney KD, Kim N. Yeast Sub1 and human PC4 are G-quadruplex binding proteins that suppress genome instability at co-transcriptionally formed G4 DNA. Nucleic Acids Res. 2017:45:5850-62.

30. Mortusewicz O, Roth W, Li N, Cardoso MC, Meisterernst M, Leonhardt H. Recruitment of RNA polymerase II cofactor PC4 to DNA damage sites. J Cell Biol. 2008;183:769-76.

31. Wang JY, Sarker AH, Cooper PK, Volkert MR. The single-strand DNA binding activity of human PC4 prevents mutagenesis and killing by oxidative DNA damage. Mol Cell Biol. 2004;24:6084-93.

32. Das C, Hizume K, Batta K, Kumar BR, Gadad SS, Ganguly S, Lorain S, Verreault A, Sadhale PP, Takeyasu K, Kundu TK. Transcriptional coactivator PC4, a chromatin-associated protein, induces chromatin condensation. Mol Cell Biol. 2006:26:8303-15.

33. Das C, Gadad SS, Kundu TK. Human positive coactivator 4 controls heterochromatinization and silencing of neural gene expression by interacting with REST/NRSF and COREST. J Mol Biol. 2010;397:1-12.

34. Shi CM, Zhu Y, Chung LWK, Su YP, Cheng TM. PC4 is a novel oncogenic gene for mesenchymal stem cell transformation and mediates the reciprocal actions between mesenchymal stem cells and prostate cancer cells. Exp Hematol. 2008:36:582-3.

35. Shi C, Mai Y, Zhu Y, Cheng T, Su Y. Spontaneous transformation of a clonal population of dermis-derived multipotent cells in culture. In Vitro Cell Dev Biol Anim. 2007:43:290-6

36. Peng Y, Yang J, Zhang E, Sun H, Wang Q, Wang T, Su Y, Shi C. Human positive coactivator 4 is a potential novel therapeutic target in non-small cell lung cancer. Cancer Gene Ther. 2012;19:690-6.

37. Chen L, Du C, Wang L, Yang C, Zhang JR, Li N, Li Y, Xie XD, Gao GD. Human positive coactivator 4 (PC4) is involved in the progression and prognosis of astrocytoma. J Neurol Sci. 2014;346:293-8.

38. Chakravarthi BV, Goswami MT, Pathi SS, Robinson AD, Cieslik M Chandrashekar DS, Agarwal S, Siddiqui J, Daignault S, Carskadon SL, et al.
MicroRNA-101 regulated transcriptional modulator SUB1 plays a role in prostate cancer. Oncogene. 2016:35:6330-40.

39. Tao S, Yu J, Xu Y, Deng B, Sun T, Hu P, Wei Z, Zhang J, Wang R, Shi C, Tan Q. PC4 induces lymphangiogenesis dependent VEGF-CNEGF-DNEGFR-3 axis activation in lung adenocarcinoma. Am J Cancer Res. 2015;5:1878-89.

40. Zhang T, Liu X, Chen X, Wang J, Wang Y, Qian D, Pang Q, Wang P. Inhibition of PC4 radiosensitizes non-small cell lung cancer by transcriptionally suppressing XLF. Cancer Med. 2018;7:1326-37.

41. Du Z, Luo Q, Yang L, Bing T, Li X, Guo W, Wu K, Zhao Y, Xiong S, Shangguan D, Wang F. Mass spectrometric proteomics reveals that nuclear protein positive cofactor PC4 selectively binds to cross-linked DNA by a trans-platinum anticancer complex. J Am Chem Soc. 2014;136:2948-51.

42. Li W, Hou JZ, Niu J, Xi ZO, Ma C, Sun H, Wang CJ, Fang D, Li Q, Xie SO. Akt inhibition promotes breast cancer metastasis through EGFR-mediated betacatenin nuclear accumulation. Cell Commun Signal. 2018;16:82.

43. van 't Veer $L J$, Dai $H$, van de Vijver MJ, He YD, Hart AA, Mao M, Peterse HL, van der Kooy K, Marton MJ, Witteveen AT, et al. Gene expression profiling predicts clinical outcome of breast cancer. Nature. 2002;415:530-6.

44. Chanrion M, Negre V, Fontaine H, Salvetat N, Bibeau F, Mac Grogan G, Mauriac L, Katsaros D, Molina F, Theillet C, Darbon JM. A gene expression signature that can predict the recurrence of tamoxifen-treated primary breast cancer. Clin Cancer Res. 2008;14:1744-52.

45. Pastushenko I, Blanpain C. EMT transition states during tumor progression and metastasis. Trends Cell Biol. 2018.

46. Pavlova NN, Thompson CB. The emerging hallmarks of Cancer metabolism. Cell Metab. 2016:23:27-47.

47. Miller DM, Thomas SD, Islam A, Muench D, Sedoris K. C-Myc and cancer metabolism. Clin Cancer Res. 2012;18:5546-53.

48. Boyle P. Triple-negative breast cancer: epidemiological considerations and recommendations. Ann Oncol. 2012;23(Suppl 6):vi7-12.

49. Crown J, O'Shaughnessy J, Gullo G. Emerging targeted therapies in triplenegative breast cancer. Ann Oncol. 2012;23(Suppl 6):vi56-65.

50. Liu H, Murphy CJ, Karreth FA, Emdal KB, White FM, Elemento O, Toker A Wulf GM, Cantley LC. Identifying and targeting sporadic oncogenic genetic aberrations in mouse models of triple-negative breast Cancer. Cancer Discov. 2018;8:354-69.

51. Fukuda A, Nakadai T, Shimada M, Tsukui T, Matsumoto M, Nogi Y, Meisterernst M, Hisatake K. Transcriptional coactivator PC4 stimulates promoter escape and facilitates transcriptional synergy by GAL4-VP16. Mol Cell Biol. 2004;24:6525-35.

52. Zhong L, Wang $Y$, Kannan $P$, Tainsky MA. Functional characterization of the interacting domains of the positive coactivator PC4 with the transcription factor AP-2a. Gene. 2003;320:155-64.

53. Ward PS, Thompson CB. Metabolic reprogramming: a cancer hallmark even Warburg did not anticipate. Cancer Cell. 2012;21:297-308

54. Gatenby RA, Gillies RJ. Why do cancers have high aerobic glycolysis? Nat Rev Cancer. 2004;4:891-9.

55. Dang CV. MYC, metabolism, cell growth, and tumorigenesis. Cold Spring Harb Perspect Med. 2013;3.

56. Palaskas N, Larson SM, Schultz N, Komisopoulou E, Wong J, Rohle D, Campos C, Yannuzzi N, Osborne JR, Linkov I, et al. 18F-fluorodeoxy-glucose positron emission tomography marks MYC-overexpressing human basal-like breast cancers. Cancer Res. 2011;71:5164-74.

57. Caforio M, Sorino C, lacovelli S, Fanciulli M, Locatelli F, Folgiero V. Recent advances in searching c-Myc transcriptional cofactors during tumorigenesis. J Exp Clin Cancer Res. 2018;37:239.

\section{Ready to submit your research? Choose BMC and benefit from:}

- fast, convenient online submission

- thorough peer review by experienced researchers in your field

- rapid publication on acceptance

- support for research data, including large and complex data types

- gold Open Access which fosters wider collaboration and increased citations

- maximum visibility for your research: over $100 \mathrm{M}$ website views per year

At BMC, research is always in progress.

Learn more biomedcentral.com/submissions 